Sādhanā Vol. 39, Part 1, February 2014, pp. 165-187. (C) Indian Academy of Sciences

\title{
Optimal way of selecting cities and conveyances for supplying coal in uncertain environment
}

\author{
AMIT KUMAR ${ }^{1}$ and AMARPREET KAUR ${ }^{2, *}$ \\ ${ }^{1}$ School of Mathematics and Computer Applications, Thapar University, \\ Patiala 147 004, India \\ ${ }^{2}$ Center for Physical and Mathematical Sciences, School of Basic and Applied \\ Sciences, Central University of Punjab, Bathinda 151 001, Punjab, India \\ e-mail: amitkdma@gmail.com; amanpreettoor@gmail.com
}

MS received 16 September 2012; revised 29 August 2013; accepted 29 August 2013

\begin{abstract}
In this paper, the limitations and shortcomings of the existing methods for solving fuzzy solid transportation problem are pointed out and to overcome these shortcomings, a new method is proposed for solving fuzzy solid transportation problem. The advantages of the proposed method over the existing methods are discussed. To illustrate the proposed method, an existing fuzzy solid transportation problem is solved. Also, to show the application of the proposed method in real life problems an existing real life fuzzy solid transportation problem is solved by the proposed method.
\end{abstract}

Keywords. Fuzzy solid transportation problem; ranking function; $L R$ flat fuzzy number.

\section{Introduction}

The traditional transportation problem is a well-known optimization problem in operational research in which two kinds of constraints are taken into consideration, i.e., source constraint and destination constraint. But, in many industrial problems, a homogeneous product is delivered from a source to a destination by means of different modes of transport called conveyances, such as trucks, cargo flights, goods trains, ships, etc. For such case, the traditional transportation problem turns into the solid transportation problem. As a generalization of traditional transportation problem, the solid transportation problem was introduced by Haley (1962).

In conventional solid transportation problems it is assumed that decision maker is sure about the precise values of all the required parameters. In real world applications, all the parameters of the transportation problems may not be known precisely due to uncontrollable factors.

To deal quantitatively with imprecise information, the concepts and techniques of probability could be employed. However, probability distributions require either apriori predictable regularity or aposteriori frequency distribution to construct. Moreover, the premise that imprecision can be equated with randomness is still questionable (Bellman \& Zadeh 1970).

${ }^{*}$ For correspondence 
As an alternative, uncertain values can be represented by membership functions of the fuzzy set theory (Zadeh 1965). The main advantages of methodologies based on fuzzy theory are that they do not require prior predictable regularities or posterior frequency distributions, and they can deal with imprecise input information containing feelings and emotions quantified based on the decision-makers subjective judgment. Due to the same reason several authors have represented the parameters of transportation problems and solid transportation problems by fuzzy numbers and proposed different methods for solving these problems.

In recent years, the solid transportation problem has received much attention and many models and algorithms under uncertain environment have been investigated. Gen et al (1995) used a genetic algorithm for solving bicriteria fuzzy solid transportation problems. Li et al (1997) proposed an improved genetic algorithm to solve multi-objective solid transportation problem with fuzzy numbers. Jimenez \& Verdegay (1997) used genetic algorithm to solve the fuzzy solid transportation problem. Jimenez \& Verdegay (1998) studied two kinds of uncertain solid transportation problem, that is, the supplies, demands and conveyance capacities are interval numbers and fuzzy numbers, respectively. Jimenez \& Verdegay (1999) designed an evolutionary algorithm based parametric approach to solve fuzzy solid transportation problem. Liu (2006) developed a solution procedure that is able to calculate the lower and upper bounds of the objective value of fuzzy solid transportation problem, where at least one of the parameters are fuzzy numbers. Yang \& Liu (2007) investigated the fixed charge solid transportation problem under fuzzy environment, in which the direct costs, the fixed charges, the supplies, the demands and the conveyance capacities are supposed to be fuzzy variables. Ojha et al (2009) formulated capacitated, multi-objective, solid transportation problems with and without entropy in fuzzy environment.

In this paper, the limitations and shortcomings of the existing methods for solving fuzzy solid transportation problem are pointed out and to overcome these limitations and shortcomings, a new method is proposed to find the fuzzy optimal solution of unbalanced fuzzy solid transportation problems.

This paper is organized as follows: In section 2, some basic definitions and arithmetic operations are presented. In section 3, the shortcomings of an existing method are discussed. In section 4, a new method is proposed to find exact fuzzy optimal solution of unbalanced fuzzy solid transportation problem. To show the application of proposed method a real life problem is solved in section 5. The conclusions are discussed in section 6.

\section{Preliminaries}

In this section, some basic definitions, arithmetic operations of $L R$ flat fuzzy numbers and Yager's ranking approach for the comparing of $L R$ flat fuzzy numbers are presented.

\subsection{Basic definitions}

In this section, some basic definitions are presented.

Definition 1 (Dubois \& Prade 1980). A fuzzy number $\widetilde{A}=(m, n, \alpha, \beta)_{L R}$ is said to be $L R$ flat fuzzy number if its membership function $\mu_{\widetilde{A}}(x)$ is given by

$$
\mu_{\tilde{A}}(x)= \begin{cases}L\left(\frac{m-x}{\alpha}\right), & \text { for } x \leq m, \alpha>0 \\ R\left(\frac{x-n}{\beta}\right), & \text { for } x \geq n, \beta>0 \\ 1, & \text { for } m \leq x \leq n .\end{cases}
$$


If $m=n$ then $\widetilde{A}=(m, n, \alpha, \beta)_{L R}$ will be converted into $\widetilde{A}=(m, \alpha, \beta)_{L R}$ and is said to be $L R$ fuzzy number.

$L$ and $R$ are called reference functions, which are continuous, non-increasing functions that defines the left and right shapes of $\mu_{\widetilde{A}}(x)$, respectively and $L(0)=R(0)=1$.

If $L(x)=R(x)=$ maximum $\{0,1-x\}$ then an $L R$ flat fuzzy number $(m, n, \alpha, \beta)_{L R}$ and an $L R$ fuzzy number $(m, \alpha, \beta)_{L R}$ are said to be trapezoidal and triangular fuzzy numbers and denoted by $(m, n, \alpha, \beta)$ and $(m, \alpha, \beta)$, respectively.

Definition 2 (Dubois \& Prade 1980). Let $\widetilde{A}=(m, n, \alpha, \beta)_{L R}$ be an $L R$ flat fuzzy number and $\lambda$ be a real number in the interval $[0,1]$ then the crisp set $A_{\lambda}=\left\{x \in X: \mu_{\tilde{A}}(x) \geq \lambda\right\}=$ $\left[m-\alpha L^{-1}(\lambda), n+\beta R^{-1}(\lambda)\right]$, is said to be $\lambda$-cut of $\widetilde{A}$.

Definition 3 (Dubois \& Prade 1980). An $L R$ flat fuzzy number $\tilde{A}=(m, n, \alpha, \beta)_{L R}$ is said to be zero $L R$ flat fuzzy number if and only if $m=0, n=0, \alpha=0, \beta=0$.

Definition 4 (Dubois \& Prade 1980). Two $L R$ flat fuzzy numbers $\tilde{A}_{1}=\left(m_{1}, n_{1}, \alpha_{1}, \beta_{1}\right)_{L R}$ and $\tilde{A}_{2}=\left(m_{2}, n_{2}, \alpha_{2}, \beta_{2}\right)_{L R}$ are said to be equal i.e., $\tilde{A}_{1}=\tilde{A}_{2}$ if and only if $m_{1}=m_{2}, n_{1}=$ $n_{2}, \alpha_{1}=\alpha_{2}, \beta_{1}=\beta_{2}$.

Definition 5 (Dehghan et al 2006). An $L R$ flat fuzzy number $\tilde{A}=(m, n, \alpha, \beta)_{L R}$ is said to be non-negative $L R$ flat fuzzy number if $m-\alpha \geq 0$.

Remark 1. If $m=n$ then an $L R$ flat fuzzy number $(m, n, \alpha, \beta)_{L R}$ is said to be an $L R$ fuzzy number and is denoted as $(m, m, \alpha, \beta)_{L R}$ or $(n, n, \alpha, \beta)_{L R}$ or $(m, \alpha, \beta)_{L R}$ or $(n, \alpha, \beta)_{L R}$.

Remark 2. If $m=n$ and $L(x)=R(x)=$ maximum $\{0,1-x\}$ then an $L R$ flat fuzzy number $(m, n, \alpha, \beta)_{L R}$ is said to be a triangular fuzzy number and is denoted as $(a, b, c)$ where, $a=$ $m-\alpha, b=m($ or $n), c=m+\beta$ (or $n+\beta)$.

Remark 3. If $m \neq n$ but $L(x)=R(x)=$ maximum $\{0,1-x\}$ then an $L R$ flat fuzzy number $(m, n, \alpha, \beta)_{L R}$ is said to be a trapezoidal fuzzy number and is denoted as $(a, b, c, d)$ where, $a=m-\alpha, b=m, c=n, d=n+\beta$.

\subsection{Arithmetic operations}

In this section, addition and multiplication operations of $L R$ flat fuzzy numbers are presented (Dubois \& Prade 1980):

(i) Let $\tilde{A}_{1}=\left(m_{1}, n_{1}, \alpha_{1}, \beta_{1}\right)_{L R}$ and $\tilde{A}_{2}=\left(m_{2}, n_{2}, \alpha_{2}, \beta_{2}\right)_{L R}$ be two $L R$ flat fuzzy numbers. Then, $\tilde{A}_{1} \oplus \tilde{A}_{2}=\left(m_{1}+m_{2}, n_{1}+n_{2}, \alpha_{1}+\alpha_{2}, \beta_{1}+\beta_{2}\right)_{L R}$.

(ii) Let $\tilde{A}_{1}=\left(m_{1}, n_{1}, \alpha_{1}, \beta_{1}\right)_{L R}$ and $\tilde{A}_{2}=\left(m_{2}, n_{2}, \alpha_{2}, \beta_{2}\right)_{L R}$ be two non-negative $L R$ flat fuzzy numbers. Then,

$$
\tilde{A}_{1} \otimes \tilde{A}_{2} \simeq\left(m_{1} m_{2}, n_{1} n_{2}, m_{1} m_{2}-\left(m_{1}-\alpha_{1}\right)\left(m_{2}-\alpha_{2}\right),\left(n_{1}+\beta_{1}\right)\left(n_{2}+\beta_{2}\right)-n_{1} n_{2}\right)_{L R} .
$$

(iii) Let $\tilde{A}_{1}=\left(m_{1}, n_{1}, \alpha_{1}, \beta_{1}\right)_{L R}$ be an $L R$ flat fuzzy number. Then,

$$
\lambda \tilde{A}_{1}=\left(\lambda m_{1}, \lambda n_{1}, \lambda \alpha_{1}, \lambda \beta_{1}\right)_{L R}, \quad \lambda \geq 0 .
$$




\subsection{Yager's ranking approach}

A number of ranking approaches has been proposed for the comparing fuzzy numbers. A relatively simple computational and easily understandable ranking approach (Yager 1981) is considered for the comparing of fuzzy numbers in this paper. Yager (1981) proposed a procedure for ordering fuzzy sets in which a ranking index $\Re(\tilde{A})$ is calculated for the fuzzy number $\widetilde{A}=$ $(m, n, \alpha, \beta)_{L R}$ from its $\lambda$-cut $A_{\lambda}=\left[m-\alpha L^{-1}(\lambda), n+\beta R^{-1}(\lambda)\right]$ according to the following formula:

$$
\Re(\tilde{A})=\frac{1}{2}\left(\int_{0}^{1}\left(m-\alpha L^{-1}(\lambda)\right) d \lambda+\int_{0}^{1}\left(n+\beta R^{-1}(\lambda)\right) d \lambda\right) .
$$

Let $\widetilde{A}$ and $\widetilde{B}$ be two $L R$ flat fuzzy numbers. Then,

(i) $\widetilde{A} \succ \widetilde{B}$ if $\Re(\widetilde{A})>\Re(\widetilde{B})$,

(ii) $\widetilde{A} \approx \widetilde{B}$ if $\Re(\widetilde{A})=\Re(\widetilde{B})$,

(iii) $\widetilde{A} \succeq \widetilde{B}$ if $\mathfrak{R}(\widetilde{A}) \geq \mathfrak{R}(\widetilde{B})$.

\section{Shortcomings and limitations of existing methods}

Several authors have proposed different methods to solve single and multi-objective solid transportation problems as well as its extensions. In this section, the shortcomings and limitations of these methods are pointed out.

\subsection{Limitations of existing methods}

(i) Since, the existing methods (Gen et al 1995; Ojha et al 2009) are proposed for solving such multi-objective solid transportation problems in which all the parameters except the quantity of the product that should be transported from source to destinations are represented by fuzzy numbers. So, these methods can also be used for solving such single-objective solid transportation problems as well as same type of single and multi-objective transportation problems. However, the existing methods (Gen et al 1995; Ojha et al 2009; Liu \& Kao 2004) can not be used to find the fuzzy optimal solution of such existing single and multi-objective transportation problems (Kumar \& Kaur 2011; Gupta et al 2011; Liu \& Kao 2004) and such single-objective solid transportation problems (Liu 2006) in which all the parameters are represented by fuzzy numbers.

(ii) Since, the existing method (Yang \& Liu 2007) is proposed to solve such single-objective fixed charge solid transportation problems in which all the parameters except the quantity of the product that should be transported from source to destinations are represented by fuzzy numbers. So, it can also be used to solve same type of fuzzy solid transportation problems and fuzzy transportation problems. However, the existing method (Yang \& Liu 2007) can not be used to find the fuzzy optimal solution of such existing single objective transportation problems (Kumar \& Kaur 2011; Liu \& Kao 2004) and single-objective solid transportation problems (Liu 2006) in which all the parameters are represented by fuzzy numbers.

(iii) Since, the existing methods (Jimenez \& Verdegay 1997, 1998, 1999) are proposed to solve such solid transportation problems in which all the parameters except cost parameters are represented by fuzzy numbers. So, these methods can also be used to find the fuzzy optimal solution of same type of single-objective transportation problems. However, the existing 
methods (Jimenez \& Verdegay 1997, 1998, 1999) can not used to find the fuzzy optimal solution of such existing single-objective transportation problems (Kumar \& Kaur 2011; Liu \& Kao 2004) and single-objective solid transportation problems (Liu 2006) in which all the parameters are represented by fuzzy numbers.

\subsection{Shortcomings of existing method}

To the best of our knowledge only the existing method (Liu 2006) can be used to solve such single-objective solid transportation problems in which all the parameters are represented by fuzzy numbers. However, it is not genuine to apply this method due to the following reason:

To show the shortcomings of the existing methods (Arenas et al 1999; Julien 1994), Liu \& Kao (2004) solved the fuzzy transportation problem, presented in Example 3.1, by using the existing methods (Arenas et al 1999; Julien 1994).

Example 3.1 (Liu \& Kao 2004): Consider a fuzzy transportation problem with inequality constraints having two sources and two destinations. Availabilities, demands and the costs are as follows:

Availabilities: $\tilde{a}_{1}=(3,1,2)_{L R}, a_{2}=5$.

Demands: $b_{1}=4, \tilde{b}_{2}=(3,2,3)_{L R}$.

Costs: $c_{11}=1, c_{12}=3, c_{21}=7, c_{22}=2$,

where, $L(x)=R(x)=$ maximum $\{0,1-x\}$.

According to Liu \& Kao (2004) the solution of fuzzy transportation problem, presented in Example 3.1, is $Z_{0}^{L}=18$ and $Z_{0}^{U}=17$, where $Z_{0}^{L}$ and $Z_{0}^{U}$ are the values of left and right end points of $\alpha$-cut of $Z$ i.e., $\left[Z_{\alpha}^{L}, Z_{\alpha}^{R}\right.$ ] at $\alpha=0$. Liu \& Kao (2004) pointed out that $Z_{0}^{L}>Z_{0}^{U}$ which contradicts the existing result $Z_{0}^{U} \geq Z_{0}^{L} \forall \alpha \in[0,1]$.

To overcome the shortcomings of the existing methods (Arenas et al 1999; Julien 1994), Liu \& Kao (2004) proposed a new method for solving fuzzy transportation problems and the same method is used for solving the fuzzy solid transportation problem (Liu 2006) and solved the fuzzy solid transportation problem, presented in Example 3.2, to illustrate his method.

Example 3.2 (Liu 2006): Consider a fuzzy solid transportation problem having two sources, three destinations and two conveyances. Fuzzy availabilities, fuzzy demands, fuzzy capacities and the costs are as follows:

Fuzzy availabilities: $\tilde{a}_{1}=(80,100,10,20)_{L R}, \tilde{a}_{2}=(70,10,20)_{L R}$.

Fuzzy demands: $\tilde{b}_{1}=(30,40,20,10)_{L R}, \tilde{b}_{2}=(50,50,10,10)_{L R}, \tilde{b}_{3}=(40,60,10,10)_{L R}$.

Fuzzy capacities: $\tilde{e}_{1}=(80,10,20)_{L R}, \tilde{e}_{2}=(70,10,20)_{L R}$.

Costs: $\tilde{c}_{111}=(30,10,10)_{L R}, c_{112}=70, c_{121}=60, c_{122}=60, c_{131}=50, c_{132}=30, \tilde{c}_{211}=$ $(20,10,10)_{L R}, c_{212}=40, c_{221}=20, c_{222}=50, c_{231}=40, c_{232}=50$.

where, $L(x)=R(x)=$ maximum $\{0,1-x\}$.

On solving the fuzzy solid transportation problem, presented in Example 3.2, by using the existing method (Liu 2006) for $\alpha=0$, the following optimal solution is obtained $x_{122}^{L}=40$ and $x_{122}^{R}=10$. Since, $x_{11}^{L}>x_{11}^{R}$ and $x_{122}^{L}>x_{122}^{R}$ so the same shortcoming, pointed out by Liu $\&$ Kao (2004) in the existing methods (Arenas et al 1999; Julien 1994), is also occurring in the existing method (Liu 2006). 


\section{Proposed method}

In this section, to overcome the limitations of the existing methods (Gen et al 1995; Jimenez \& Verdegay 1997, 1998, 1999; Ojha et al 2009; Yang \& Liu 2007) and to resolve the shortcomings of the existing method (Liu 2006), discussed in section 3, a new method, based on tabular representation, is proposed to find the fuzzy optimal solution of fuzzy solid transportation problem having $p$ sources, $q$ destinations and $k$ conveyances. Let the fuzzy availability of the product at $i$ th source $\left(S_{i}\right)$, fuzzy demand of the product at $j$ th destination $\left(D_{j}\right)$, fuzzy capacity of the $k$ th conveyance $\left(E_{k}\right)$ i.e., the maximum quantity of the product which can be carried by the $k$ th conveyance and the fuzzy cost for transporting one unit quantity of the product from $i$ th source to $j$ th destination by means of the $k$ th conveyance be represented by $L R$ flat fuzzy numbers $\tilde{a}_{i}, \tilde{b}_{j}$, $\tilde{e}_{k}$ and $\tilde{c}_{i j k}$, respectively.

The steps of the proposed method are as follows:

Step 1: Find $\sum_{i=1}^{p} \tilde{a}_{i}, \sum_{j=1}^{q} \tilde{b}_{j}$ and $\sum_{k=1}^{r} \tilde{e}_{k}$. Let $\sum_{i=1}^{p} \tilde{a}_{i}=(m, n, \alpha, \beta)_{L R}, \sum_{j=1}^{q} \tilde{b}_{j}=\left(m^{\prime}, n^{\prime}, \alpha^{\prime}, \beta^{\prime}\right)_{L R}$ and $\sum_{k=1}^{r} \tilde{e}_{k}=\left(m^{\prime \prime}, n^{\prime \prime}, \alpha^{\prime \prime}, \beta^{\prime \prime}\right)_{L R}$. Use Definition 4 to examine that the problem is balanced or unbalanced.

Case (1): If the problem is balanced, i.e., $\sum_{i=1}^{p} \tilde{a}_{i}=\sum_{j=1}^{q} \tilde{b}_{j}=\sum_{k=1}^{r} \tilde{e}_{k}$, then Go to Step 4.

Case (2): If the problem is unbalanced i.e., $\sum_{i=1}^{p} \tilde{a}_{i}=\sum_{j=1}^{q} \tilde{b}_{j} \neq \sum_{k=1}^{r} \tilde{e}_{k}$ or $\sum_{i=1}^{p} \tilde{a}_{i} \neq \sum_{j=1}^{q} \tilde{b}_{j}=$ $\sum_{k=1}^{r} \tilde{e}_{k}$ or $\sum_{i=1}^{p} \tilde{a}_{i}=\sum_{k=1}^{r} \tilde{e}_{k} \neq \sum_{j=1}^{q} \tilde{b}_{j}$ or $\sum_{i=1}^{p} \tilde{a}_{i} \neq \sum_{j=1}^{q} \tilde{b}_{j} \neq \sum_{k=1}^{r} \tilde{e}_{k}$ then Go to Step 2.

Step 2: Check that $\sum_{i=1}^{p} \tilde{a}_{i}=\sum_{j=1}^{q} \tilde{b}_{j}$ or $\sum_{i=1}^{p} \tilde{a}_{i} \neq \sum_{j=1}^{q} \tilde{b}_{j}$.

Case (1): If $\sum_{i=1}^{p} \tilde{a}_{i}=\sum_{j=1}^{q} \tilde{b}_{j}$ then Go to Step 3.

Case (2): If $\sum_{i=1}^{p} \tilde{a}_{i} \neq \sum_{j=1}^{q} \tilde{b}_{j}$ then convert $\sum_{i=1}^{p} \tilde{a}_{i} \neq \sum_{j=1}^{q} \tilde{b}_{j}$ into $\sum_{i=1}^{u} \tilde{a}_{i}=\sum_{j=1}^{t} \tilde{b}_{j}$, where, $u=p$ or $p+1$ and $t=q$ or $q+1$ by using one of the following cases:

Case (2a): If $m-\alpha \leq m^{\prime}-\alpha^{\prime}, \alpha \leq \alpha^{\prime}, n-m \leq n^{\prime}-m^{\prime}$ and $\beta \leq \beta^{\prime}$ then introduce a dummy source $S_{p+1}$ with fuzzy availability $\tilde{a}_{p+1}=\left(m^{\prime}-m, n^{\prime}-n, \alpha^{\prime}-\alpha\right.$, $\left.\beta^{\prime}-\beta\right)_{L R}$ so that $\sum_{i=1}^{p+1} \tilde{a}_{i}=\sum_{j=1}^{q} \tilde{b}_{j}$. Go to Step 3 .

Case (2b): If $m-\alpha \geq m^{\prime}-\alpha^{\prime}, \alpha \geq \alpha^{\prime}, n-m \geq n^{\prime}-m^{\prime}$ and $\beta \geq \beta^{\prime}$ then introduce a dummy destination $D_{q+1}$ with fuzzy demand $\tilde{b}_{q+1}=\left(m-m^{\prime}, n-n^{\prime}, \alpha-\right.$ $\left.\alpha^{\prime}, \beta-\beta^{\prime}\right)_{L R}$ so that $\sum_{i=1}^{p} \tilde{a}_{i}=\sum_{j=1}^{q+1} \tilde{b}_{j}$. Go to Step 3.

Case (2c): If neither Case (2a) nor Case (2b) is satisfied then introduce a dummy source $S_{p+1}$ with fuzzy availability $\tilde{a}_{p+1}=\left(\operatorname{maximum}\left\{0,\left(m^{\prime}-\alpha^{\prime}\right)-(m-\alpha)\right\}+\right.$ 
maximum $\left\{0,\left(\alpha^{\prime}-\alpha\right)\right\}$, maximum $\left\{0,\left(m^{\prime}-\alpha^{\prime}\right)-(m-\alpha)\right\}+$ maximum $\left\{0,\left(\alpha^{\prime}-\alpha\right)\right\}+$ maximum $\left\{0,\left(n^{\prime}-m^{\prime}\right)-(n-m)\right\}$, maximum $\left\{0,\left(\alpha^{\prime}-\right.\right.$ $\alpha)\}$, maximum $\left.\left\{0,\left(\beta^{\prime}-\beta\right)\right\}\right)_{L R}$ and a dummy destination $D_{q+1}$ with fuzzy demand $\tilde{b}_{q+1}=\left(\operatorname{maximum}\left\{0,(m-\alpha)-\left(m^{\prime}-\alpha^{\prime}\right)\right\}+\operatorname{maximum}\{0,(\alpha-\right.$ $\left.\left.\alpha^{\prime}\right)\right\}$, maximum $\left\{0,(m-\alpha)-\left(m^{\prime}-\alpha^{\prime}\right)\right\}+\operatorname{maximum}\left\{0,\left(\alpha-\alpha^{\prime}\right)\right\}+$ maximum $\left\{0,(n-m)-\left(n^{\prime}-m^{\prime}\right)\right\}$, maximum $\left\{0,\left(\alpha-\alpha^{\prime}\right)\right\}$, maximum $\left.\left\{0,\left(\beta-\beta^{\prime}\right)\right\}\right)_{L R}$ so that $\sum_{i=1}^{p+1} \tilde{a}_{i}=\sum_{j=1}^{q+1} \tilde{b}_{j}$. Go to Step 3 .

Step 3: Using Step 2, $\sum_{i=1}^{u} \tilde{a}_{i}=\sum_{j=1}^{t} \tilde{b}_{j}$, where $u=p$ or $p+1$ and $t=q$ or $q+1$.

Let $\sum_{i=1}^{u} \tilde{a}_{i}=\sum_{j=1}^{t} \tilde{b}_{j}=\left(m_{1}, n_{1}, \alpha_{1}, \beta_{1}\right)_{L R}$ and $\sum_{k=1}^{r} \tilde{e}_{k}=\left(m^{\prime \prime}, n^{\prime \prime}, \alpha^{\prime \prime}, \beta^{\prime \prime}\right)_{L R}$

Now check $\sum_{i=1}^{u} \tilde{a}_{i}=\sum_{j=1}^{t} \tilde{b}_{j}=\sum_{k=1}^{r} \tilde{e}_{k}$ or $\sum_{i=1}^{u} \tilde{a}_{i}=\sum_{j=1}^{t} \tilde{b}_{j} \neq \sum_{k=1}^{r} \tilde{e}_{k}$.

Case (1): If $\sum_{i=1}^{u} \tilde{a}_{i}=\sum_{j=1}^{t} \tilde{b}_{j}=\sum_{k=1}^{r} \tilde{e}_{k}$, then Go to Step 4.

Case (2): If $\sum_{i=1}^{u} \tilde{a}_{i}=\sum_{j=1}^{t} \tilde{b}_{j} \neq \sum_{k=1}^{r} \tilde{e}_{k}$ then convert $\sum_{i=1}^{u} \tilde{a}_{i}=\sum_{j=1}^{t} \tilde{b}_{j} \neq \sum_{k=1}^{r} \tilde{e}_{k}$ into $\sum_{i=1}^{m} \tilde{a}_{i}=$ $\sum_{j=1}^{n} \tilde{b}_{j}=\sum_{k=1}^{l} \tilde{e}_{k}$, where $m=p$ or $p+1, n=q$ or $q+1$ and $l=r$ or $r+1$ by using one of the following cases:

Case (2a): If $m_{1}-\alpha_{1} \leq m^{\prime \prime}-\alpha^{\prime \prime}, \alpha_{1} \leq \alpha^{\prime \prime}, n_{1}-m_{1} \leq n^{\prime \prime}-m^{\prime \prime}$, and $\beta_{1} \leq \beta^{\prime \prime}$ then check that in Step 2 a dummy source $S_{p+1}$ with fuzzy availability $\tilde{a}_{p+1}$ is introduced or not and also check that a dummy destination $D_{q+1}$ with fuzzy demand $\tilde{b}_{q+1}$ is introduced or not.

Case (i): If both the dummy source $S_{p+1}$ and dummy destination $D_{q+1}$ are introduced then increase both the fuzzy availability $\tilde{a}_{p+1}$ of the already introduced dummy source $S_{p+1}$ and the fuzzy demand of the already introduced dummy destination $D_{q+1}$ by the same fuzzy quantity $\tilde{a}=\left(m^{\prime \prime}-m_{1}, n^{\prime \prime}-n_{1}, \alpha^{\prime \prime}-\alpha_{1}, \beta^{\prime \prime}-\beta_{1}\right)_{L R}$ i.e., replace fuzzy availability $\tilde{a}_{p+1}$ and fuzzy demand $\tilde{b}_{q+1}$ of the already introduced dummy source $S_{p+1}$ and destinations $D_{q+1}$ by $\tilde{a}_{p+1} \oplus \tilde{a}$ and $\tilde{b}_{q+1} \oplus \tilde{a}$ so that $\sum_{i=1}^{p+1} \tilde{a}_{i}=\sum_{j=1}^{q+1} \tilde{b}_{j}=\sum_{k=1}^{r} \tilde{e}_{k}$. Go to Step 4.

Case (ii): If a dummy source $S_{p+1}$ with fuzzy availability $\tilde{a}_{p+1}$ is introduced but no dummy destination $D_{q+1}$ with fuzzy demand $\tilde{b}_{q+1}$ is introduced then increase the fuzzy availability $\tilde{a}_{p+1}$ of the already introduced dummy source $S_{p+1}$ by the fuzzy quantity $\tilde{a}=\left(m^{\prime \prime}-\right.$ $\left.m_{1}, n^{\prime \prime}-n_{1}, \alpha^{\prime \prime}-\alpha_{1}, \beta^{\prime \prime}-\beta_{1}\right)_{L R}$ i.e., replace fuzzy availability $\tilde{a}_{p+1}$ of the already introduced dummy source $S_{p+1}$ by $\tilde{a}_{p+1} \oplus \tilde{a}$ and also introduce a dummy destination with fuzzy demand $\tilde{b}_{q+1}=\tilde{a}=$ 


$$
\begin{aligned}
& \left(m^{\prime \prime}-m_{1}, n^{\prime \prime}-n_{1}, \alpha^{\prime \prime}-\alpha_{1}, \beta^{\prime \prime}-\beta_{1}\right)_{L R} \text { so that } \sum_{i=1}^{p+1} \tilde{a}_{i}=\sum_{j=1}^{q+1} \tilde{b}_{j}= \\
& \sum_{k=1}^{r} \tilde{e}_{k} \text {. Go to Step } 4 .
\end{aligned}
$$

Case (iii): If a dummy destination $D_{q+1}$ with fuzzy demand $\tilde{b}_{q+1}$ is introduced but no dummy source $S_{p+1}$ with fuzzy availability $\tilde{a}_{p+1}$ is introduced then increase the fuzzy demand $\tilde{b}_{q+1}$ of the already introduced dummy destination $D_{q+1}$ by the fuzzy quantity $\tilde{a}=$ $\left(m^{\prime \prime}-m_{1}, n^{\prime \prime}-n_{1}, \alpha^{\prime \prime}-\alpha_{1}, \beta^{\prime \prime}-\beta_{1}\right)_{L R}$ i.e., replace fuzzy demand $\tilde{b}_{q+1}$ of the already introduced dummy destination $D_{q+1}$ by $\tilde{b}_{q+1} \oplus \tilde{a}$ and also introduce a dummy source $S_{p+1}$ with fuzzy availability $\tilde{a}_{p+1}=\tilde{a}=\left(m^{\prime \prime}-m_{1}, n^{\prime \prime}-n_{1}, \alpha^{\prime \prime}-\alpha_{1}, \beta^{\prime \prime}-\beta_{1}\right)_{L R}$ so that $\sum_{i=1}^{p+1} \tilde{a}_{i}=\sum_{j=1}^{q+1} \tilde{b}_{j}=\sum_{k=1}^{r} \tilde{e}_{k}$. Go to Step 4.

Case (2b): If $m_{1}-\alpha_{1} \geq m^{\prime \prime}-\alpha^{\prime \prime}, \alpha_{1} \geq \alpha^{\prime \prime}, n_{1}-m_{1} \geq n^{\prime \prime}-m^{\prime \prime}$, and $\beta_{1} \geq \beta^{\prime \prime}$ then introduce a dummy conveyance $E_{r+1}$ with fuzzy capacity $\tilde{e}_{r+1}=\left(m_{1}-\right.$ $\left.m^{\prime \prime}, n_{1}-n^{\prime \prime}, \alpha_{1}-\alpha^{\prime \prime}, \beta_{1}-\beta^{\prime \prime}\right)_{L R}$ so that $\sum_{i=1}^{u} \tilde{a}_{i}=\sum_{j=1}^{t} \tilde{b}_{j}=\sum_{k=1}^{r+1} \tilde{e}_{k}$. Go to Step 5.

Case (2c): If neither Case (2a) nor Case (2b) then check that in Step 2 a dummy source $S_{p+1}$ with fuzzy availability $\tilde{a}_{p+1}$ is introduced or not and also check that a dummy destination $D_{q+1}$ with fuzzy demand $\tilde{b}_{q+1}$ is introduced or not.

Case (i): If both the dummy source $S_{p+1}$ and dummy destination $D_{q+1}$ are introduced then increase both the fuzzy availability $\tilde{a}_{p+1}$ of the already introduced dummy source $S_{p+1}$ and the fuzzy demand of the already introduced dummy destination $D_{q+1}$ by the same fuzzy quantity $\tilde{a}=$ (maximum $\left\{0,\left(m^{\prime \prime}-\alpha^{\prime \prime}\right)-\left(m_{1}-\alpha_{1}\right)\right\}+$ maximum $\left\{0,\left(\alpha^{\prime \prime}-\alpha_{1}\right)\right\}$, maximum $\left\{0,\left(m^{\prime \prime}-\alpha^{\prime \prime}\right)-\left(m_{1}-\alpha_{1}\right)\right\}+\max -$ imum $\left\{0,\left(\alpha^{\prime \prime}-\alpha_{1}\right)\right\}+$ maximum $\left\{0,\left(n^{\prime \prime}-m^{\prime \prime}\right)-\left(n_{1}-m_{1}\right)\right\}$, maximum $\left\{0,\left(\alpha^{\prime \prime}-\alpha_{1}\right)\right\}$, maximum $\left.\left\{0,\left(\beta^{\prime \prime}-\beta_{1}\right)\right\}\right)_{L R}$ i.e., replace fuzzy availability $\tilde{a}_{p+1}$ and fuzzy demand $\tilde{b}_{q+1}$ of the already introduced dummy source $S_{p+1}$ and destinations $D_{q+1}$ by $\tilde{a}_{p+1} \oplus \tilde{a}$ and $\tilde{b}_{q+1} \oplus \tilde{a}$. Also, introduce a dummy conveyance $E_{r+1}$ with fuzzy capacity $\tilde{e}_{r+1}=\left(\operatorname{maximum}\left\{0,\left(m_{1}-\alpha_{1}\right)-\left(m^{\prime \prime}-\alpha^{\prime \prime}\right)\right\}+\right.$ maximum $\left\{0,\left(\alpha_{1}-\alpha^{\prime \prime}\right)\right\}$, maximum $\left\{0,\left(m_{1}-\alpha_{1}\right)-\left(m^{\prime \prime}-\alpha^{\prime \prime}\right)\right\}+$ maximum $\left\{0,\left(\alpha_{1}-\alpha^{\prime \prime}\right)\right\}+\operatorname{maximum}\left\{0,\left(n_{1}-m_{1}\right)-\left(n^{\prime \prime}-m^{\prime \prime}\right)\right\}$, maxi$\operatorname{mum}\left\{0,\left(\alpha_{1}-\alpha^{\prime \prime}\right)\right\}$, maximum $\left.\left\{0,\left(\beta_{1}-\beta^{\prime \prime}\right)\right\}\right)_{L R}$ so that $\sum_{i=1}^{p+1} \tilde{a}_{i}=$ $\sum_{j=1}^{q+1} \tilde{b}_{j}=\sum_{k=1}^{r+1} \tilde{e}_{k}$. Go to Step 4.

Case (ii): If a dummy source $S_{p+1}$ with fuzzy availability $\tilde{a}_{p+1}$ is introduced but no dummy destination $D_{q+1}$ with fuzzy demand $\tilde{b}_{q+1}$ is introduced then increase the fuzzy availability $\tilde{a}_{p+1}$ of the already introduced dummy source $S_{p+1}$ by the fuzzy quantity $\tilde{a}=$ (maximum $\left\{0,\left(m^{\prime \prime}-\alpha^{\prime \prime}\right)-\left(m_{1}-\alpha_{1}\right)\right\}+\operatorname{maximum}\left\{0,\left(\alpha^{\prime \prime}-\alpha_{1}\right)\right\}$, maximum $\{0$, $\left.\left(m^{\prime \prime}-\alpha^{\prime \prime}\right)-\left(m_{1}-\alpha_{1}\right)\right\}+$ maximum $\left\{0,\left(\alpha^{\prime \prime}-\alpha_{1}\right)\right\}+$ maximum 
$\left\{0,\left(n^{\prime \prime}-m^{\prime \prime}\right)-\left(n_{1}-m_{1}\right)\right\}$, maximum $\left\{0,\left(\alpha^{\prime \prime}-\alpha_{1}\right)\right\}$, maximum $\left.\left\{0,\left(\beta^{\prime \prime}-\beta_{1}\right)\right\}\right)_{L R}$ i.e., replace fuzzy availability $\tilde{a}_{p+1}$ of the already introduced dummy source $S_{p+1}$ by $\tilde{a}_{p+1} \oplus \tilde{a}$ and also introduce a dummy destination with fuzzy demand $\tilde{b}_{q+1}=\tilde{a}=$ (maximum $\{0$, $\left.\left(m^{\prime \prime}-\alpha^{\prime \prime}\right)-\left(m_{1}-\alpha_{1}\right)\right\}+$ maximum $\left\{0,\left(\alpha^{\prime \prime}-\alpha_{1}\right)\right\}$, maximum $\left\{0,\left(m^{\prime \prime}-\alpha^{\prime \prime}\right)-\left(m_{1}-\alpha_{1}\right)\right\}+\operatorname{maximum}\left\{0,\left(\alpha^{\prime \prime}-\alpha_{1}\right)\right\}+\operatorname{maximum}\{0$, $\left.\left(n^{\prime \prime}-m^{\prime \prime}\right)-\left(n_{1}-m_{1}\right)\right\}$, maximum $\left\{0,\left(\alpha^{\prime \prime}-\alpha_{1}\right)\right\}$, maximum $\left.\left\{0,\left(\beta^{\prime \prime}-\beta_{1}\right)\right\}\right)_{L R}$. Also, introduce a dummy conveyance $E_{r+1}$ with fuzzy capacity $\tilde{e}_{r+1}=\left(\right.$ maximum $\left\{0,\left(m_{1}-\alpha_{1}\right)-\left(m^{\prime \prime}-\alpha^{\prime \prime}\right)\right\}+$ maximum $\left\{0,\left(\alpha_{1}-\alpha^{\prime \prime}\right)\right\}$, maximum $\left\{0,\left(m_{1}-\alpha_{1}\right)-\left(m^{\prime \prime}-\alpha^{\prime \prime}\right)\right\}+$ maximum $\left\{0,\left(\alpha_{1}-\alpha^{\prime \prime}\right)\right\}+$ maximum $\left\{0,\left(n_{1}-m_{1}\right)-\left(n^{\prime \prime}-m^{\prime \prime}\right)\right\}$, maximum $\left\{0,\left(\alpha_{1}-\alpha^{\prime \prime}\right)\right\}$, maximum $\left.\left\{0,\left(\beta_{1}-\beta^{\prime \prime}\right)\right\}\right)_{L R}$ so that $\sum_{i=1}^{p+1} \tilde{a}_{i}=\sum_{j=1}^{q+1} \tilde{b}_{j}=$ $\sum_{k=1}^{r+1} \tilde{e}_{k}$. Go to Step 4.

Case (iii): If a dummy destination $D_{q+1}$ with fuzzy demand $\tilde{b}_{q+1}$ is introduced but no dummy source $S_{p+1}$ with fuzzy availability $\tilde{a}_{p+1}$ is introduced then increase the fuzzy demand $\tilde{b}_{q+1}$ of the already introduced dummy destination $D_{q+1}$ by the fuzzy quantity $\tilde{a}=$ (maximum $\left\{0,\left(m^{\prime \prime}-\alpha^{\prime \prime}\right)-\left(m_{1}-\alpha_{1}\right)\right\}+\operatorname{maximum}\left\{0,\left(\alpha^{\prime \prime}-\right.\right.$ $\left.\left.\alpha_{1}\right)\right\}$, maximum $\left\{0,\left(m^{\prime \prime}-\alpha^{\prime \prime}\right)-\left(m_{1}-\alpha_{1}\right)\right\}+$ maximum $\left\{0,\left(\alpha^{\prime \prime}-\alpha_{1}\right)\right\}+$ maximum $\left\{0,\left(n^{\prime \prime}-m^{\prime \prime}\right)-\left(n_{1}-m_{1}\right)\right\}$, maximum $\left\{0,\left(\alpha^{\prime \prime}-\alpha_{1}\right)\right\}$, maximum $\left.\left\{0,\left(\beta^{\prime \prime}-\beta_{1}\right)\right\}\right)_{L R}$ i.e., replace fuzzy demand $\tilde{b}_{q+1}$ of the already introduced dummy destination $D_{q+1}$ by $\tilde{b}_{q+1} \oplus \tilde{a}$ and also introduced a dummy source $S_{p+1}$ with fuzzy availability $\tilde{a}_{p+1}=\tilde{a}=$ (maximum $\left\{0, a^{\prime \prime}-a_{1}\right\}$, maximum (maximum $\left\{0,\left(m^{\prime \prime}-\alpha^{\prime \prime}\right)-\left(m_{1}-\alpha_{1}\right)\right\}+$ maximum $\left\{0,\left(\alpha^{\prime \prime}-\alpha_{1}\right)\right\}$, maximum $\left\{0,\left(m^{\prime \prime}-\alpha^{\prime \prime}\right)-\left(m_{1}-\alpha_{1}\right)\right\}+$ maximum $\left\{0,\left(\alpha^{\prime \prime}-\alpha_{1}\right)\right\}+\operatorname{maximum}\left\{0,\left(n^{\prime \prime}-m^{\prime \prime}\right)-\left(n_{1}-m_{1}\right)\right\}$, maximum $\left\{0,\left(\alpha^{\prime \prime}-\alpha_{1}\right)\right\}$, maximum $\left.\left\{0,\left(\beta^{\prime \prime}-\beta_{1}\right)\right\}\right)_{L R}$. Also, introduce a dummy conveyance $E_{r+1}$ with fuzzy capacity $\tilde{e}_{r+1}=$ (maximum $\left\{0,\left(m_{1}-\alpha_{1}\right)-\left(m^{\prime \prime}-\alpha^{\prime \prime}\right)\right\}+$ maximum $\left\{0,\left(\alpha_{1}-\alpha^{\prime \prime}\right)\right\}$, maxi$\operatorname{mum}\left\{0,\left(m_{1}-\alpha_{1}\right)-\left(m^{\prime \prime}-\alpha^{\prime \prime}\right)\right\}+\operatorname{maximum}\left\{0,\left(\alpha_{1}-\alpha^{\prime \prime}\right)\right\}+$ $\operatorname{maximum}\left\{0,\left(n_{1}-m_{1}\right)-\left(n^{\prime \prime}-m^{\prime \prime}\right)\right\}$, maximum $\left\{0,\left(\alpha_{1}-\alpha^{\prime \prime}\right)\right\}$, $\left.\operatorname{maximum}\left\{0,\left(\beta_{1}-\beta^{\prime \prime}\right)\right\}\right)_{L R}$ so that $\sum_{i=1}^{p+1} \tilde{a}_{i}=\sum_{j=1}^{q+1} \tilde{b}_{j}=\sum_{k=1}^{r+1} \tilde{e}_{k}$. Go to Step 4.

Step 4: Represent the balanced fuzzy solid transportation problem, obtained from Step 3, into tabular form as shown by table 1 by assuming the following fuzzy transportation costs $\tilde{c}_{i j k}=\left(m_{i j k}^{\prime}, n_{i j k}^{\prime}, \alpha_{i j k}^{\prime}, \beta_{i j k}^{\prime}\right)_{L R}$ as zero $L R$ flat fuzzy numbers:

(i) If it is required to add any dummy source then assume the fuzzy cost for transporting one unit quantity of the product from the introduced dummy source to all destinations by all conveyances as zero $L R$ flat fuzzy number.

(ii) If it is required to add any dummy destination then assume the fuzzy cost for transporting one unit quantity of the product from all sources to the introduced dummy destination by all conveyances as zero $L R$ flat fuzzy number. 
174

Amit Kumar and Amarpreet Kaur

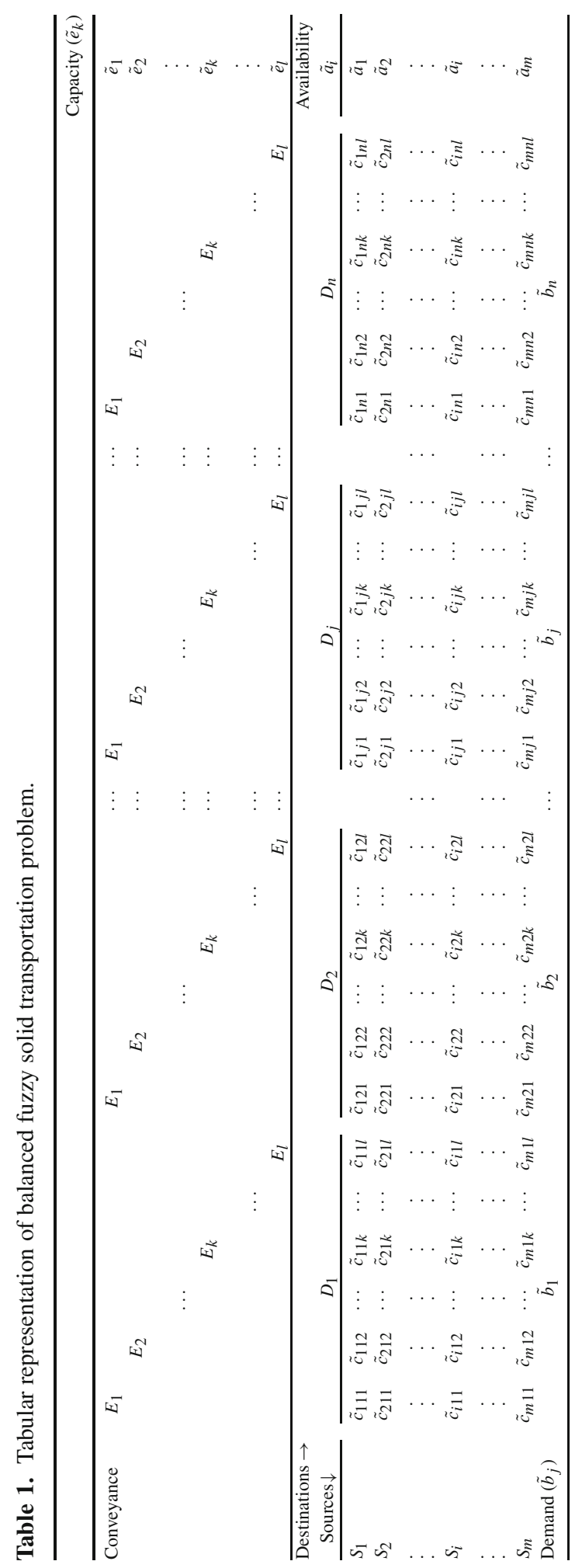


(iii) If it is required to add any dummy conveyance then assume the fuzzy cost for transporting one unit quantity of the product from the all sources to all destinations by introduced dummy conveyance as zero $L R$ flat fuzzy number.

Step 5: Split table 1 into four crisp transportation tables i.e., tables 2-5. The cost for transporting one unit quantity of the product from $i$ th source to $j$ th destination by means of $k$ th conveyance in tables 2, 3, 4 and 5 are represented by $\eta_{i j k}, \rho_{i j k}, \delta_{i j k}$ and $\xi_{i j k}$, respectively,

where,

$$
\begin{aligned}
\eta_{i j k}= & \frac{1}{2}\left(\left(m_{i j k}^{\prime}+n_{i j k}^{\prime}\right)-\alpha_{i j k}^{\prime} \int_{0}^{1} L^{-1}(\lambda) d \lambda+\beta_{i j k}^{\prime} \int_{0}^{1} R^{-1}(\lambda) d \lambda\right), \\
& i=1,2, \ldots, m, j=1,2, \ldots, n \text { and }, k=1,2, \ldots, l \\
\rho_{i j k}= & \frac{1}{2}\left(\left(m_{i j k}^{\prime}+n_{i j k}^{\prime}\right)-m_{i j k}^{\prime} \int_{0}^{1} L^{-1}(\lambda) d \lambda+\beta_{i j k}^{\prime} \int_{0}^{1} R^{-1}(\lambda) d \lambda\right), \\
& i=1,2, \ldots, m, j=1,2, \ldots, n \text { and }, k=1,2, \ldots, l \\
\delta_{i j k}= & \frac{1}{2}\left(n_{i j k}^{\prime}+\beta_{i j k}^{\prime} \int_{0}^{1} R^{-1}(\lambda) d \lambda\right), \\
\xi_{i j k}= & \frac{1}{2}\left(\left(n_{i j k}^{\prime}+\beta_{i j k}^{\prime}\right) \int_{0}^{1} R^{-1}(\lambda) d \lambda\right), \\
& i=1,2, \ldots, m, j=1,2, \ldots, n \text { and }, k=1,2, \ldots, l .
\end{aligned}
$$

Step 6: Solve the crisp solid transportation problems shown by tables 2-5 by using the existing method (Haley 1962) to find the optimal solution $\left\{m_{i j k}-\alpha_{i j k}\right\} ;\left\{\alpha_{i j k}\right\} ;\left\{n_{i j k}-m_{i j k}\right\}$ and $\left\{\beta_{i j k}\right\}$, respectively.

Step 7: Solve the equations, obtained in Step 6, to find the values of $m_{i j k}, n_{i j k}, \alpha_{i j k}$ and $\beta_{i j k}$.

Step 8: Find the fuzzy optimal solution $\left\{\tilde{x}_{i j k}\right\}$ by putting the values of $m_{i j k}, n_{i j k}, \alpha_{i j k}, \beta_{i j k}$ in $\tilde{x}_{i j k}=\left(m_{i j k}, n_{i j k}, \alpha_{i j k}, \beta_{i j k}\right)_{L R}$.

Step 9: Find the minimum total fuzzy transportation cost by putting the values of $\tilde{x}_{i j k}$ in $\sum_{i=1}^{m} \sum_{j=1}^{n} \sum_{k=1}^{l} \tilde{c}_{i j k} \otimes \tilde{x}_{i j k}$.

\subsection{Advantages of the proposed methods}

In this section, the advantages of the proposed method, over the existing methods (Gen et al 1995; Jimenez \& Verdegay 1997, 1998, 1999; Liu 2006; Ojha et al 2009; Yang \& Liu 2007) are discussed.

(i) As discussed in section 3, none of the existing methods (Gen et al 1995; Jimenez \& Verdegay 1997, 1998, 1999; Ojha et al 2009; Yang \& Liu 2007), can be used to solve such solid transportation problems in which all the parameters are represented by fuzzy numbers while the proposed method can be used to solve such solid transportation problems as well as the fuzzy solid transportation problem which can be solved by the existing methods (Gen et al 1995; Jimenez \& Verdegay 1997, 1998, 1999; Ojha et al 2009; Yang \& Liu 2007). 


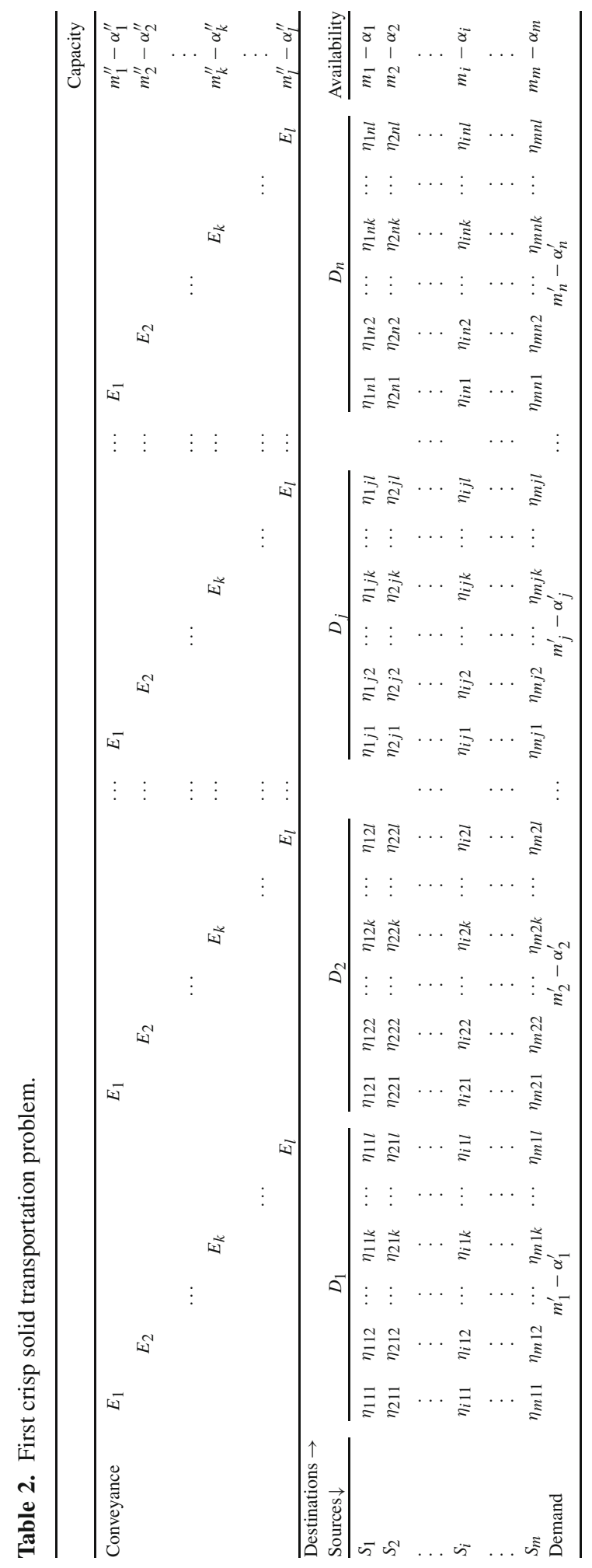




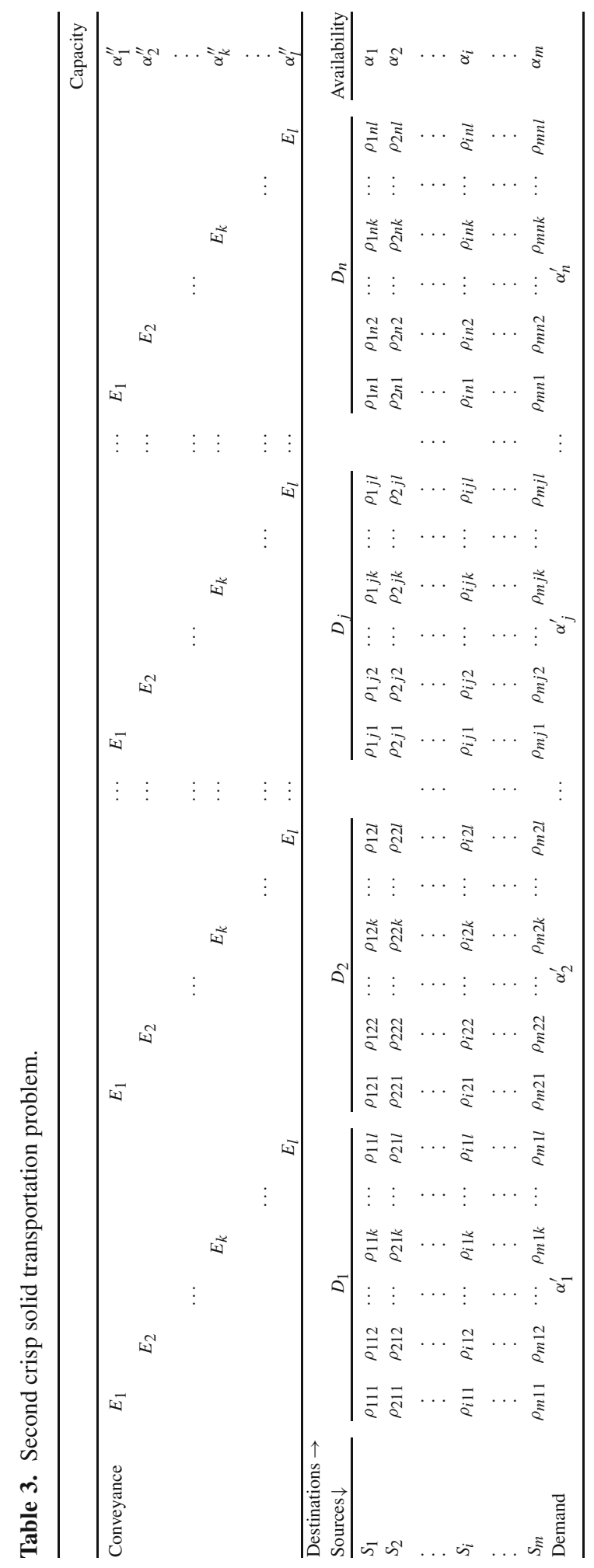




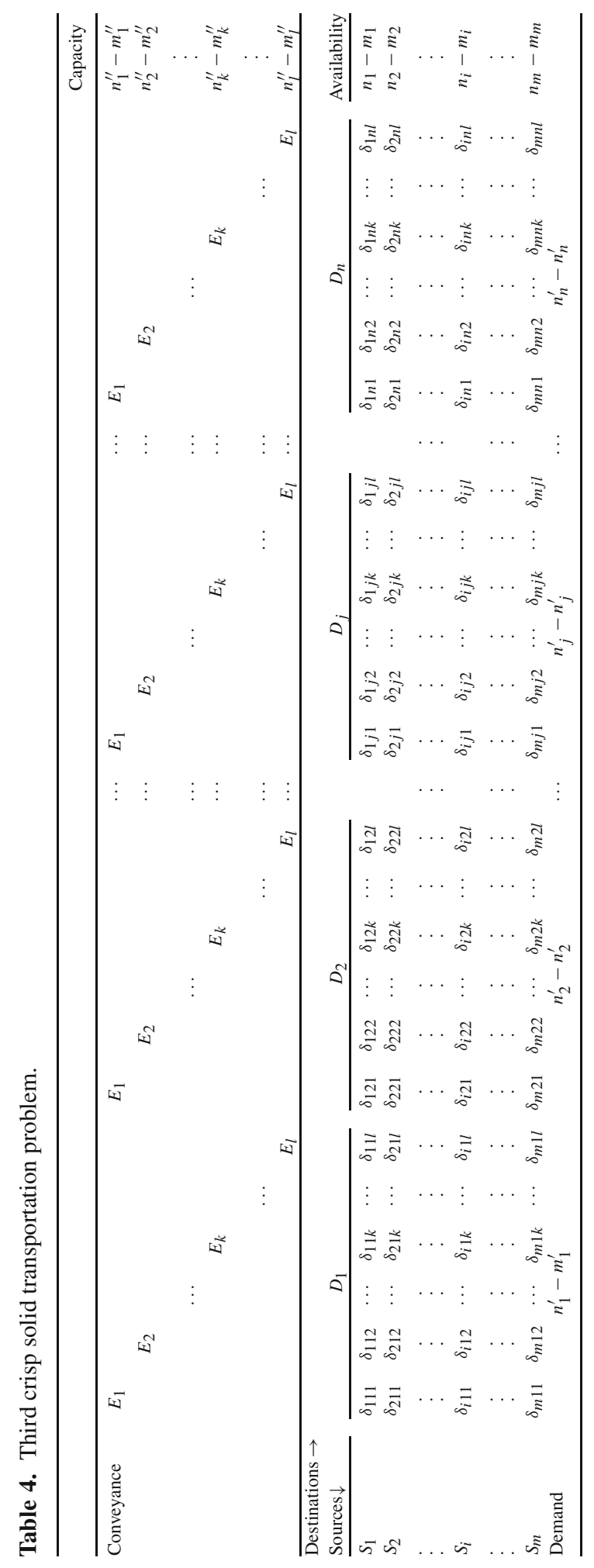




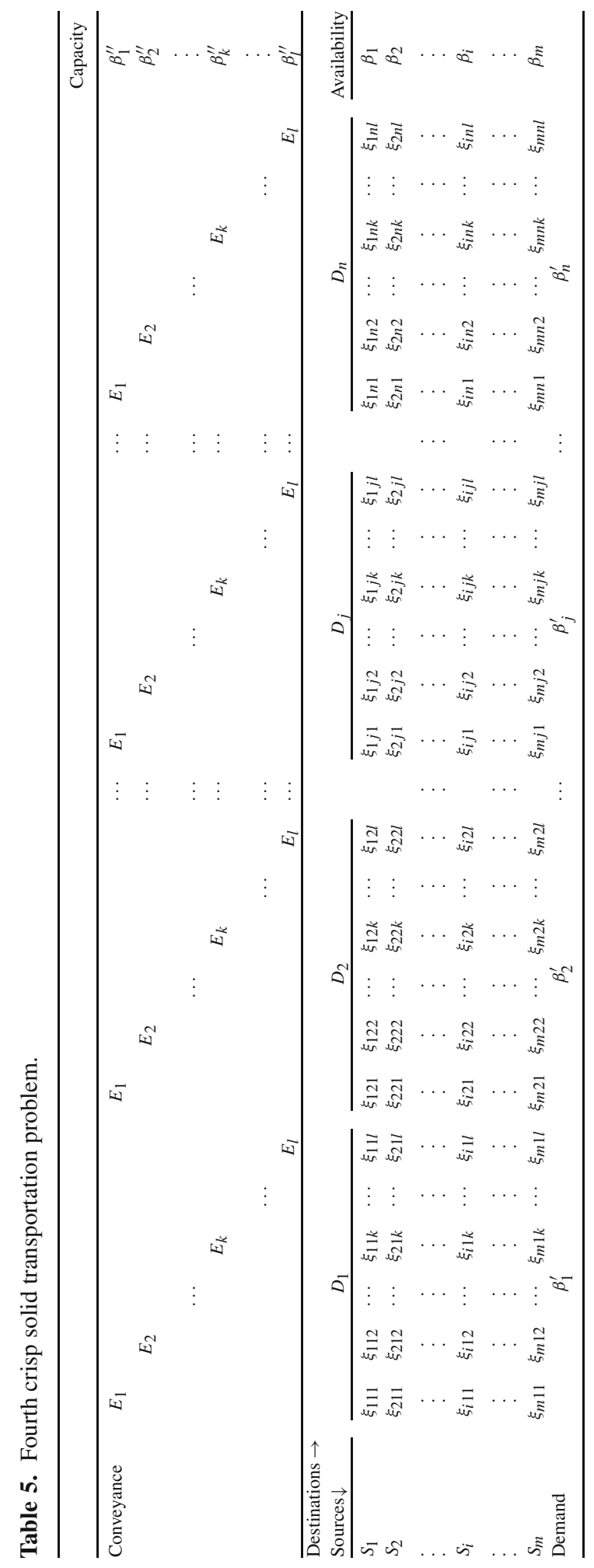


(ii) Since, in the proposed methods the restrictions $b-a \geq 0, c-b \geq 0$ and $d-c \geq 0$ are used and due to these restrictions, the restriction $d \geq a$ (or $x^{R} \geq x^{L}$ ) will always be satisfied so, by using the proposed methods the shortcomings of the existing method (Liu 2006), pointed out in section 3.2, is resolved.

\subsection{Illustrative example}

In this section, to illustrate the proposed methods, the existing fuzzy solid transportation problem, presented in Example 3.2 is solved by the proposed method.

4.2a Fuzzy optimal solution of the chosen problem: Using the proposed method, the fuzzy optimal solution of the fuzzy solid transportation problem, chosen in Example 3.2, can be obtained as follows:

Step 1: Using values of $\tilde{a}_{1}, \tilde{a}_{2}, \tilde{b}_{1}, \tilde{b}_{2}, \tilde{b}_{3}, \tilde{e}_{1}$ and $\tilde{e}_{2}$, total fuzzy availability $\sum_{i=1}^{2} \tilde{a}_{i}=$ $(150,170,20,40)_{L R}$, total fuzzy demand $\sum_{j=1}^{3} \tilde{b}_{j}=(120,150,40,30)_{L R}$ and total fuzzy capacity $\sum_{k=1}^{2} \tilde{e}_{k}=(150,150,20,40)_{L R}$. Since $\sum_{i=1}^{2} \tilde{a}_{i} \neq \sum_{j=1}^{3} \tilde{b}_{j} \neq \sum_{k=1}^{2} \tilde{e}_{k}$, so it is an unbalanced fuzzy solid transportation problem.

Step 2: Comparing $\sum_{i=1}^{2} \tilde{a}_{i}=(150,170,20,40)_{L R}$ by $\sum_{i=1}^{p} \tilde{a}_{i}=(m, n, \alpha, \beta)_{L R}$ and $\sum_{j=1}^{3} \tilde{b}_{j}=$ $(120,150,40,30)_{L R}$ by $\sum_{j=1}^{q} \tilde{b}_{j}=\left(m^{\prime}, n^{\prime}, \alpha^{\prime}, \beta^{\prime}\right)_{L R}$ the values of $m, n, \alpha, \beta, m^{\prime}, n^{\prime}, \alpha^{\prime}$ and $\beta^{\prime}$ are $150,170,20,40,120,150,40$ and 30, respectively.

Since, $\sum_{i=1}^{2} \tilde{a}_{i} \neq \sum_{j=1}^{3} \tilde{b}_{j}$ and neither the condition $m_{1}-\alpha_{1} \leq m^{\prime}-\alpha^{\prime}, \alpha_{1} \leq \alpha^{\prime}, n_{1}-$ $m_{1} \leq n^{\prime}-m^{\prime}, \beta_{1} \leq \beta^{\prime}$ nor the condition $m_{1}-\alpha_{1} \geq m^{\prime}-\alpha^{\prime}, \alpha_{1} \geq \alpha^{\prime}, n_{1}-m_{1} \geq n^{\prime}-m^{\prime}$, $\beta_{1} \geq \beta^{\prime}$ is satisfied so, as described in Case (2c) of Step 2 of the proposed method, there is need to introduce a dummy source $S_{3}$ with fuzzy availability $\tilde{a}_{3}=(20,30,20,0)_{L R}$ and a dummy destination $D_{4}$ with fuzzy demand $\tilde{b}_{4}=(50,50,0,10)_{L R}$ so that $\sum_{i=1}^{3} \tilde{a}_{i}=$ $\sum_{j=1}^{4} \tilde{b}_{j}$

Step 3: Using Step 2, $\sum_{i=1}^{3} \tilde{a}_{i}=\sum_{j=1}^{4} \tilde{b}_{j}=(170,200,40,40)_{L R}$. Since, $\sum_{i=1}^{3} \tilde{a}_{i}=\sum_{j=1}^{4} \tilde{b}_{j} \neq \sum_{k=1}^{2} \tilde{e}_{k}$ so Go to Step 4 of the proposed method.

Comparing $\sum_{i=1}^{3} \tilde{a}_{i}=\sum_{j=1}^{4} \tilde{b}_{j}=(170,200,40,40)_{L R}$ by $\sum_{i=1}^{3} \tilde{a}_{i}=\sum_{j=1}^{4} \tilde{b}_{j}=\left(m_{1}, n_{1}\right.$, $\left.\alpha_{1}, \beta_{1}\right)_{L R}$ and $\sum_{k=1}^{2} \tilde{e}_{k}=(150,150,20,40)_{L R}$ by $\sum_{k=1}^{r} \tilde{e}_{k}=\left(m^{\prime \prime}, n^{\prime \prime}, \alpha^{\prime \prime}, \beta^{\prime \prime}\right)_{L R}$ the values of $m_{1}, n_{1}, \alpha_{1}, \beta_{1}, m^{\prime \prime}, n^{\prime \prime}, \alpha^{\prime \prime}$ and $\beta^{\prime \prime}$ are 170, 200, 40, 40, 150, 150, 20 and 40, respectively. 


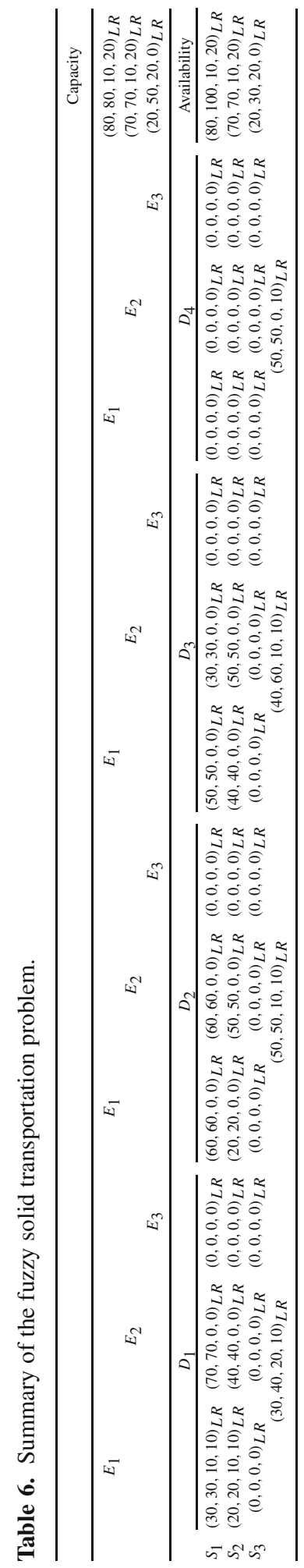


Table 7. First crisp solid transportation problem.

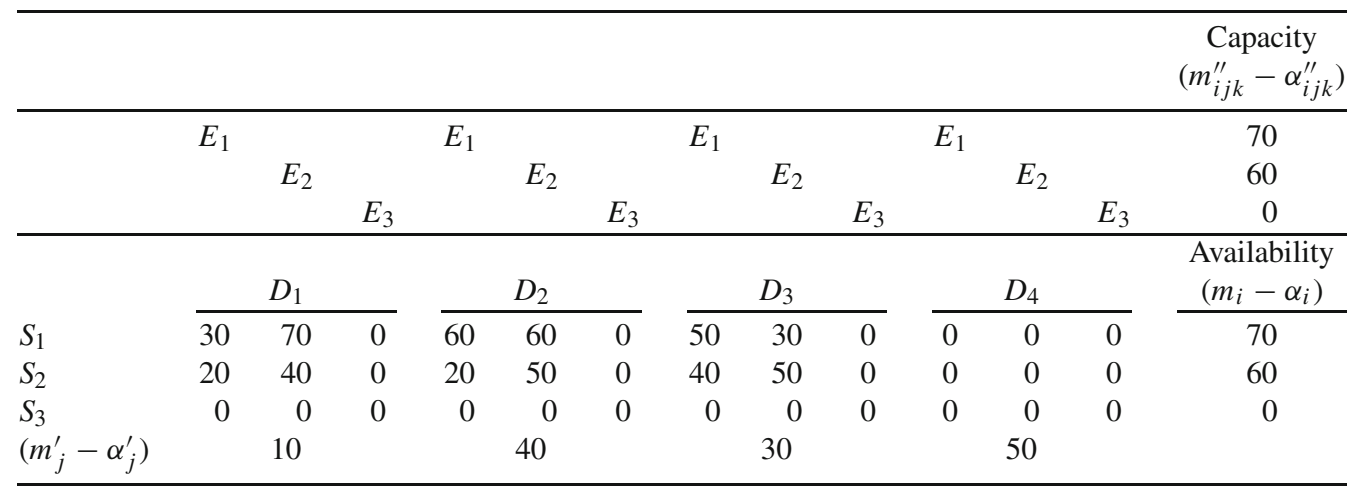

Since, the condition $m_{1} \geq m^{\prime \prime}, m_{1}-\alpha_{1} \geq m^{\prime \prime}-\alpha^{\prime \prime}, \alpha_{1} \geq \alpha^{\prime \prime}, n_{1}-m_{1} \geq n^{\prime \prime}-m^{\prime \prime}$ is satisfied so as described in Case (2b) of Step 3 of the proposed method there is need to introduce a dummy conveyance $E_{3}$ with fuzzy capacity $\tilde{e}_{3}=(20,50,20,0)_{L R}$ so that $\sum_{i=1}^{3} \tilde{a}_{i}=\sum_{j=1}^{4} \tilde{b}_{j}=\sum_{k=1}^{3} \tilde{e}_{k}$.

Step 4: The balanced fuzzy solid transportation problem, obtained from Step 3, can be represented by table 6 .

Step 5: Using Step 5 of the proposed method, table 6 can be split into four crisp solid transportation tables i.e., tables 7-10.

Step 6: The optimal solution of crisp solid transportation problems, shown by tables 7-10, are $m_{113}-\alpha_{113}=0, m_{123}-\alpha_{123}=0, m_{132}-\alpha_{132}=30, m_{133}-\alpha_{133}=0, m_{141}-\alpha_{141}=$ $20, m_{142}-\alpha_{142}=20, m_{211}-\alpha_{211}=10, m_{221}-\alpha_{221}=40, m_{242}-\alpha_{242}=10, m_{213}-$ $\alpha_{213}=0, m_{223}-\alpha_{223}=0, m_{311}-\alpha_{311}=0, m_{313}-\alpha_{313}=0, m_{332}-\alpha_{332}=0 ; \alpha_{113}=$ $5, \alpha_{123}=5, \alpha_{132}=0, \alpha_{133}=0, \alpha_{141}=0, \alpha_{142}=0, \alpha_{211}=0, \alpha_{221}=0, \alpha_{242}=0$, $\alpha_{213}=5, \alpha_{223}=5, \alpha_{311}=10, \alpha_{313}=0, \alpha_{332}=10 ; n_{113}-m_{113}=0, n_{123}-m_{123}=$ $0, n_{132}-m_{132}=0, n_{133}-m_{133}=20, n_{141}-m_{141}=0, n_{142}-m_{142}=0, n_{211}-m_{211}=$ $0, n_{221}-m_{221}=0, n_{242}-m_{242}=0, n_{213}-m_{213}=0, n_{223}-m_{223}=0, n_{311}-m_{311}=$ $0, n_{313}-m_{313}=10, n_{332}-m_{332}=0$ and $\beta_{132}=10, \beta_{142}=10, \beta_{211}=10, \beta_{221}=10$, respectively.

Table 8. Second crisp solid transportation problem.

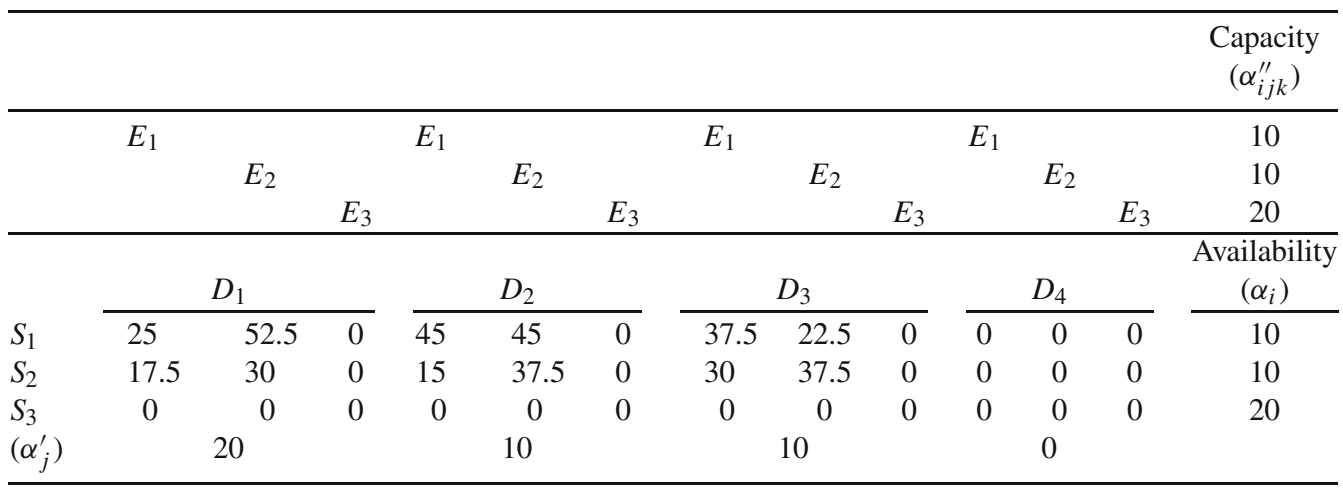


Table 9. Third crisp solid transportation problem.

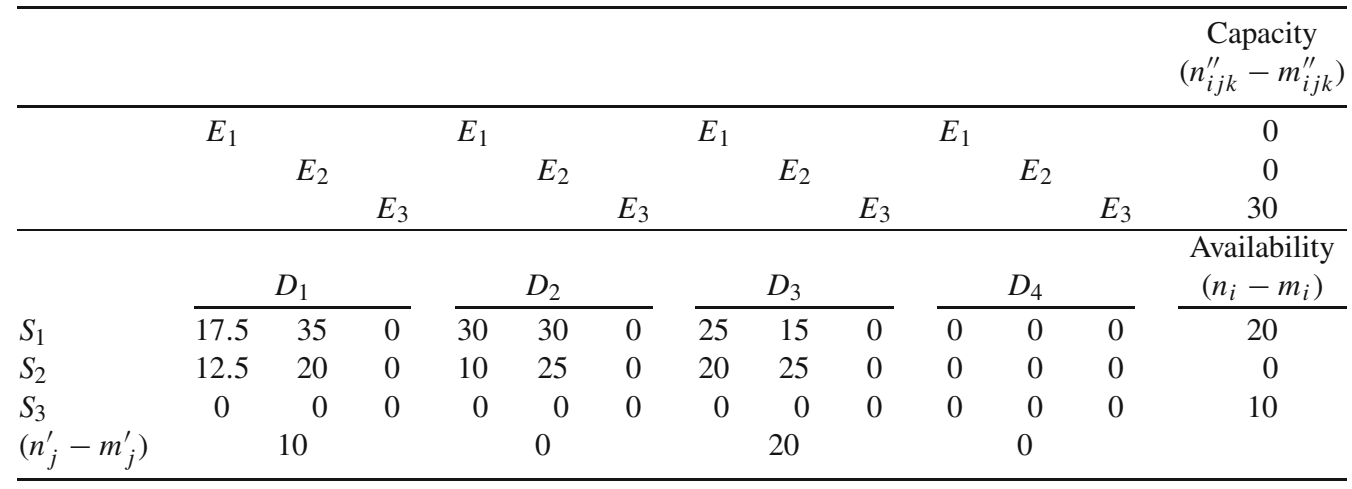

Step 7: On solving the equations, obtained from Step 6, the values of $m_{i j k}, n_{i j k}, \alpha_{i j k}$ and $\beta_{i j k}$ are $m_{113}=5, n_{113}=5, \alpha_{113}=5, \beta_{113}=0, m_{123}=5, n_{123}=5, \alpha_{123}=5, \beta_{123}=0$, $m_{132}=30, n_{132}=30, \alpha_{132}=0, \beta_{132}=10, m_{133}=0, n_{133}=20, \alpha_{133}=0, \beta_{133}=0$, $m_{141}=20, n_{141}=20, \alpha_{141}=0, \beta_{141}=0, m_{142}=20, n_{142}=20, \alpha_{142}=0, \beta_{142}=$ $10, m_{211}=10, n_{211}=10, \alpha_{211}=0, \beta_{211}=10, m_{221}=40, n_{221}=40, \alpha_{221}=$ $0, \beta_{221}=10, m_{242}=10, n_{242}=10, \alpha_{242}=0, \beta_{242}=0, m_{213}=5, n_{213}=5, \alpha_{213}=$ $5, \beta_{213}=0, m_{223}=5, n_{223}=5, \alpha_{223}=5, \beta_{223}=0, m_{311}=10, n_{311}=10, \alpha_{311}=$ $10, \beta_{311}=0, m_{313}=0, n_{313}=10, \alpha_{313}=0, \beta_{313}=0, m_{332}=10, n_{332}=10, \alpha_{332}=$ $10, \beta_{332}=0$ and remaining values of $m_{i j k}, n_{i j k}, \alpha_{i j k}, \beta_{i j k}$ are zero, respectively.

Step 8: Putting the values of $m_{i j k}, n_{i j k}, \alpha_{i j k}$ and $\beta_{i j k}$ in $\tilde{x}_{i j k}=\left(m_{i j k}, n_{i j k}, \alpha_{i j k}, \beta_{i j k}\right)_{L R}$, the fuzzy optimal solution is $\tilde{x}_{113}=(5,5,5,0)_{L R}, \tilde{x}_{123}=(5,5,5,0)_{L R}$, $\tilde{x}_{132}=(30,30,0,10)_{L R}, \tilde{x}_{133}=(0,20,0,0)_{L R}, \tilde{x}_{141}=(20,20,0,0)_{L R}, \tilde{x}_{142}=$ $(20,20,0,10)_{L R}, \tilde{x}_{211}=(10,10,0,10)_{L R}, \tilde{x}_{221}=(40,40,0,10)_{L R}, \tilde{x}_{242}=$ $(10,10,0,0)_{L R}, \tilde{x}_{213}=(5,5,5,0)_{L R}, \tilde{x}_{223}=(5,5,5,0)_{L R}, \tilde{x}_{311}=(10,10,10,0)_{L R}$, $\tilde{x}_{313}=(0,10,0,0)_{L R}, \tilde{x}_{332}=(10,10,10,0)_{L R}$ and remaining values of $\tilde{x}_{i j k}$ are zero, respectively.

Step 9: Putting the values of $\tilde{x}_{111}, \tilde{x}_{112}, \tilde{x}_{113}, \tilde{x}_{121}, \tilde{x}_{122}, \tilde{x}_{123}, \tilde{x}_{131}, \tilde{x}_{132}, \tilde{x}_{133}, \tilde{x}_{141}, \tilde{x}_{142}, \tilde{x}_{143}$, $\tilde{x}_{211}, \tilde{x}_{212}, \tilde{x}_{213}, \tilde{x}_{221}, \tilde{x}_{222}, \tilde{x}_{223}, \tilde{x}_{231}, \tilde{x}_{232}, \tilde{x}_{233}, \tilde{x}_{241}, \tilde{x}_{242}, \tilde{x}_{243}, \tilde{x}_{311}, \tilde{x}_{312}, \tilde{x}_{313}, \tilde{x}_{321}$,

Table 10. Fourth crisp solid transportation problem.

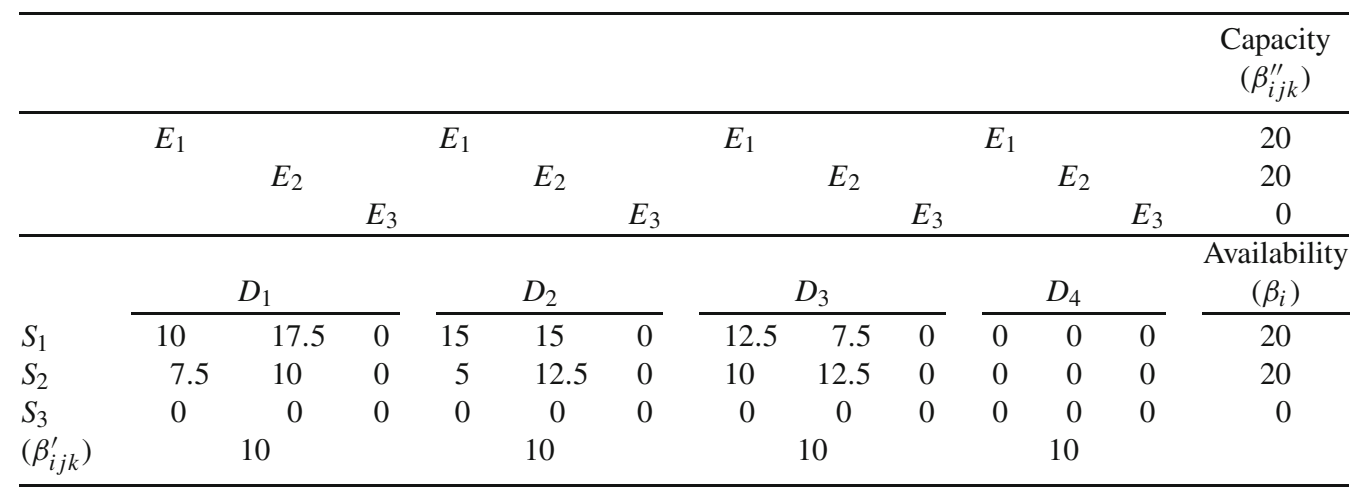


$\tilde{x}_{322}, \tilde{x}_{323}, \tilde{x}_{331}, \tilde{x}_{332}, \tilde{x}_{333}, \tilde{x}_{341}, \tilde{x}_{342}, \tilde{x}_{343}$ in $\left((30,30,10,10)_{L R} \otimes \tilde{x}_{111} \oplus 70 \tilde{x}_{112} \oplus\right.$ $(0,0,0,0)_{L R} \otimes \tilde{x}_{113} \oplus 60 \tilde{x}_{121} \oplus 60 \tilde{x}_{122} \oplus(0,0,0,0)_{L R} \otimes \tilde{x}_{123} \oplus 50 \tilde{x}_{131} \oplus 30 \tilde{x}_{132} \oplus$ $(0,0,0,0)_{L R} \otimes \tilde{x}_{133} \oplus(0,0,0,0)_{L R} \otimes \tilde{x}_{141} \oplus(0,0,0,0)_{L R} \otimes \tilde{x}_{142} \oplus(0,0,0,0)_{L R} \otimes$ $\tilde{x}_{143} \oplus(20,20,10,10)_{L R} \otimes \tilde{x}_{211} \oplus 40 \tilde{x}_{212} \oplus(0,0,0,0)_{L R} \otimes \tilde{x}_{213} \oplus 20 \tilde{x}_{221} \oplus 50 \tilde{x}_{222} \oplus$ $(0,0,0,0)_{L R} \otimes \tilde{x}_{223} \oplus 40 \tilde{x}_{231} \oplus 50 \tilde{x}_{232} \oplus(0,0,0,0)_{L R} \otimes \tilde{x}_{233} \oplus(0,0,0,0)_{L R} \otimes \tilde{x}_{241} \oplus$ $(0,0,0,0)_{L R} \otimes \tilde{x}_{242} \oplus(0,0,0,0)_{L R} \otimes \tilde{x}_{243} \oplus(0,0,0,0)_{L R} \otimes \tilde{x}_{311} \oplus(0,0,0,0)_{L R} \otimes$ $\tilde{x}_{312} \oplus(0,0,0,0)_{L R} \otimes \tilde{x}_{313} \oplus(0,0,0,0)_{L R} \otimes \tilde{x}_{321} \oplus(0,0,0,0)_{L R} \otimes \tilde{x}_{322} \oplus$ $(0,0,0,0)_{L R} \otimes \tilde{x}_{323} \oplus(0,0,0,0)_{L R} \otimes \tilde{x}_{331} \oplus(0,0,0,0)_{L R} \otimes \tilde{x}_{332} \oplus(0,0,0,0)_{L R} \otimes$ $\left.\tilde{x}_{333} \oplus(0,0,0,0)_{L R} \otimes \tilde{x}_{341} \oplus(0,0,0,0)_{L R} \otimes \tilde{x}_{342} \oplus(0,0,0,0)_{L R} \otimes \tilde{x}_{343}\right)$ the minimum total fuzzy transportation cost is $(1900,1900,100,900)_{L R}$.

4.2b Physical interpretation of the results: In this section, the minimum total fuzzy transportation cost, obtained by using the proposed methods, is physically interpreted. Similarly, the obtained fuzzy optimal solutions $\tilde{x}_{i j}$ can also be physically interpreted.

Using the proposed methods, the minimum total fuzzy transportation cost is (1900, 1900, 100, 900), which can be physically interpreted as follows:

(i) The least amount of minimum total transportation cost is 1800 .

(ii) The most possible amount of minimum total transportation cost is 1900 .

(iii) The greatest amount of minimum total transportation cost is 2800 .

i.e., the minimum total transportation cost will be always greater than 1800 and less than 2800 and maximum chances of the minimum total transportation cost be 1900 .

\section{Case study}

Yang \& Liu (2007) proposed a method to find the crisp optimal solution of fuzzy fixed charge solid transportation problems and used it to find the crisp optimal solution of real life fuzzy fixed charge solid transportation problem described in section 5.1.

To show the application of the proposed method, the fuzzy optimal solution of the same real life problem by assuming that there is no fixed charge are obtained with the help of proposed method.

\subsection{Description of problem}

As we know, coal is a kind of crucial energy source in the development of economy and society. Accordingly, how to transport the coal from mines to the different areas economically is also an important issue in the coal transportation. For the convenience of description, the problem can be summarized as follows. Suppose that there are two coal mines to supply the coal for two cities. During the process of transportation, two kinds of conveyances are available to be selected, i.e., train and cargo ship. Now, the task for the decision-maker is to make the transportation plan for the next month. At the beginning of this task, the decision maker needs to obtain the basic data, such as supply capacity, demand, transportation capacity, transportation cost of unit product, and so on. In fact, since the transportation plan is made in advance, we generally cannot get these data exactly. For this condition, the usual way is to obtain the fuzzy data by means of experience evaluation or expert advice. In this example, the notations $\tilde{a}_{i}, \tilde{b}_{j}$ and $\tilde{e}_{k}$ are employed to denote 
Table 11. The direct cost by train.

\begin{tabular}{lcccc}
\hline Cities $\rightarrow$ & 1 & 2 & 3 & 4 \\
\cline { 2 - 5 } mines $\downarrow$ & \multicolumn{5}{c}{} \\
\hline 1 & $(5,8,2,2)_{L R}$ & $(8,9,1,1)_{L R}$ & $(17,19,2,1)_{L R}$ & $(15,17,2,2)_{L R}$ \\
2 & $(7,8,2,1)_{L R}$ & $(9,10,3,1)_{L R}$ & $(4,6,1,1)_{L R}$ & $(20,23,4,2)_{L R}$ \\
3 & $(8,9,3,1)_{L R}$ & $(15,17,2,2)_{L R}$ & $(6,8,3,1)_{L R}$ & $(8,11,1,2)_{L R}$ \\
4 & $(19,21,1,3)_{L R}$ & $(12,13,3,2)_{L R}$ & $(10,11,3,3)_{L R}$ & $(10,13,1,2)_{L R}$ \\
\hline
\end{tabular}

the availability, demand and transportation capacity, respectively. The corresponding fuzzy data, with $L(x)=R(x)=$ maximum $\{0,1-x\}$ are listed as follows:

$$
\begin{array}{llll}
\tilde{a}_{1}=(28,29,3,2)_{L R}, & \tilde{a}_{2}=(20,23,4,2)_{L R}, & \tilde{a}_{3}=(34,36,2,2)_{L R}, & \tilde{a}_{4}=(30,32,2,2)_{L R}, \\
\tilde{b}_{1}=(13,14,1,1)_{L R}, & \tilde{b}_{2}=(23,26,3,1)_{L R}, & \tilde{b}_{3}=(21,23,2,1)_{L R}, & \tilde{b}_{4}=(27,29,2,2)_{L R}, \\
\tilde{e}_{1}=(45,50,6,5)_{L R}, & \tilde{e}_{2}=(65,70,5,5)_{L R} . &
\end{array}
$$

For the same reason, the transportation cost of unit amount accurately in advance can not be obtained, and it can also be treated as a fuzzy variable by experience or expert advice. For this example, the transportation cost of unit amount is listed in tables 11 and 12 .

Now, the aim is to find how much quantity of the product should be transported from which coal mine to which city by which conveyance so that the total fuzzy transportation cost is minimum.

\subsection{Results}

On solving the chosen real life problem by using the proposed method, the obtained fuzzy optimal solution $\tilde{x}_{i j k}$, representing the fuzzy quantity of the coal, that should be transported from $i$ th coal mine to $j$ th city by $k$ th conveyance to minimize the total fuzzy transportation cost and the fuzzy optimal value, representing the minimum total fuzzy transportation cost are $\tilde{x}_{111}=(7,7,0,0)_{L R}, \tilde{x}_{121}=(16,17,3,0)_{L R}, \tilde{x}_{231}=(2,3,2,0)_{L R}, \tilde{x}_{331}=$ $(19,20,0,0)_{L R}, \tilde{x}_{122}=(5,5,0,0)_{L R}, \tilde{x}_{242}=(18,20,2,0)_{L R}, \tilde{x}_{342}=(9,9,0,0), \tilde{x}_{412}=$ $(6,7,1,0), \tilde{x}_{352}=(5,6,1,0), \tilde{x}_{423}=(2,2,0,0), \tilde{x}_{452}=(22,23,1,0), \tilde{x}_{351}=(1,1,1,0)$, $\tilde{x}_{521}=(0,2,0,0), \tilde{x}_{152}=(0,0,0,2), \tilde{x}_{213}=(0,0,0,1), \tilde{x}_{252}=(0,0,0,1), \tilde{x}_{343}=$ $(0,0,0,2), \tilde{x}_{451}=(0,0,0,2), \tilde{x}_{511}=(0,0,0,3), \tilde{x}_{522}=(0,0,0,1), \tilde{x}_{532}=(0,0,0,1)$ and $(540,750,214,129)_{L R}$, respectively.

Table 12. The direct cost by Cargo ship.

\begin{tabular}{lcccc}
\hline $\begin{array}{l}\text { Cities } \rightarrow \\
\text { mines } \downarrow\end{array}$ & 1 & 2 & 3 & 4 \\
\cline { 2 - 5 } & \multicolumn{5}{c}{} & & \\
\hline 1 & $(9,12,2,1)_{L R}$ & $(9,10,4,2)_{L R}$ & $(12,13,3,2)_{L R}$ & $(25,26,5,2)_{L R}$ \\
2 & $(12,14,2,1)_{L R}$ & $(14,16,2,2)_{L R}$ & $(17,18,6,2)_{L R}$ & $(5,8,2,2)_{L R}$ \\
3 & $(10,12,2,2)_{L R}$ & $(23,25,3,2)_{L R}$ & $(25,27,2,2)_{L R}$ & $(8,10,2,2)_{L R}$ \\
4 & $(8,9,2,1)_{L R}$ & $(28,30,2,2)_{L R}$ & $(32,33,2,2)_{L R}$ & $(32,38,2,2)_{L R}$ \\
\hline
\end{tabular}




\subsection{Physical interpretation of the results}

In this section, the minimum total fuzzy transportation cost, obtained by using the proposed method, is physically interpreted. Similarly, the obtained fuzzy optimal solutions $\tilde{x}_{i j k}$ can also be physically interpreted.

Using the proposed method the minimum total fuzzy transportation cost is $(540,750,214,129)_{L R}$, which can be physically interpreted as follows:

(i) The least amount of minimum total transportation cost is 226 units.

(ii) The most possible amount of minimum total transportation cost lies between 540 and 750 units.

(iii) The greatest amount of minimum total transportation is 879 units.

i.e., the minimum total transportation cost will be always greater than 226 units and less than 879 units and maximum chances are that the minimum total transportation cost will lie between 540 and 750 units.

Remark 4. Since, the chosen real life problem, is an unbalanced problem so, to find the solution of this problem a dummy source (5), a dummy destination (5) and dummy conveyance (3) is introduced. In the results, presented in section 5.2, $\tilde{x}_{5 j k}, \tilde{x}_{i 5 k}$ and $\tilde{x}_{i j 3}$ represents the fuzzy quantity of the product that should be transported from purely dummy source (5) to $j$ th destination by means of $k$ th conveyance, $i$ th source to purely dummy destination (5) by means of $k$ th conveyance and $i$ th source to $j$ th destination by means of dummy conveyance (3), respectively.

\section{Conclusions}

On the basis of the proposed study, it can be concluded that all the problems which can be solved by using the other methods can also be solved by the proposed method. Also, there can exist several problems which can be solved by the proposed method, but can not be solved by using existing methods. Hence, it is better to use the proposed method as compared to the existing methods.

The proposed method can neither be used to find the fuzzy optimal solution of multi-objective fuzzy solid transshipment problems nor to find the single and multi-objective fixed charge solid transportation problems (Ojha et al 2010, 2011). In future, it can be tried to modify the proposed method to find fuzzy optimal solution of these problems.

\section{Acknowledgements}

The authors would like to thank the Editor-in-Chief and the anonymous reviewers for their suggestions which have led to an improvement in the quality and clarity of the paper.

\section{References}

Arenas Parra M, Bilbao Terol A and Rodriguez Uria M V 1999 Solving the multiobjective possibilistic linear programming problem. Eur. J. Operational Res. 117: 79-90

Bellman R E and Zadeh L A 1970 Decision-making in a fuzzy environment. Management Sci. 17: 141-164

Dehghan M, Hashemi B and Ghatee M 2006 Computational methods for solving fully fuzzy linear systems. Appl. Math. Comput. 179: 328-343

Dubois D and Prade H 1980 Fuzzy sets and systems: Theory and applications. Academic Press, New York 
Gen M, Ida K, Li Y and Kubota E 1995 Solving bicriteria solid transportation problem with fuzzy numbers by a genetic algorithm. Comput. Ind. Eng. 29: 537-541

Haley K B 1962 New methods in mathematical programming - The solid transportation problem. Oper. Res. 11: 448-463

Jimenez F and Verdegay J L 1997 Obtaining fuzzy solutions to the fuzzy solid transportation problem with genetic algorithms. Proc. Sixth IEEE Internat. Conf. Fuzzy Systems, Barcelona, Spain, 1657-1663

Jimenez F and Verdegay J L 1998 Uncertain solid transportation problems. Fuzzy Sets and Systems 100: $45-57$

Jimenez F and Verdegay J L 1999 Solving fuzzy solid transportation problems by an evolutionary algorithm based parametric approach. Eur. J. Operational Res. 117: 485-510

Julien B 1994 An extension to possibilistic linear programming. Fuzzy Sets and Systems 64: 195-206

Kumar A and Kaur A 2011 Application of classical transportation methods to find the fuzzy optimal solution of fuzzy transportation problems. Fuzzy Information and Eng. 3: 81-99

Gupta A, Kumar A and Kaur A 2012 Mehar's method to find exact fuzzy optimal solution of unbalanced fully fuzzy multi-objective transportation problems. Optimization Letters 6: 1737-1751

Li Y, Ida K and Gen M 1997 Improved genetic algorithm for solving multiobjective solid transportation problem with fuzzy numbers. Comput. Ind. Eng. 33: 589-592

Liu S T 2006 Fuzzy total transportation cost measures for fuzzy solid transportation problem. Appl. Math. Comput. 174: 927-941

Liu S T and Kao C 2004 Solving fuzzy transportation problems based on extension principle. Eur. J. Operational Res. 153: 661-674

Ojha A, Das B, Mondala S and Maiti M 2009 An entropy based solid transportation problem for general fuzzy costs and time with fuzzy equality. Math. Comput. Model. 50: 166-178

Ojha A, Das B, Mondala S and Maiti M 2010 A solid transportation problem for an item with fixed charge, vehicle cost and price discounted varying charge using genetic algorithm. Appl. Soft Computing 10: $100-110$

Ojha A, Mondala S and Maiti M 2011 Transportation policies for single and multi objective transportation problem using fuzzy logic. Math. Comput. Model. 53: 1637-1646

Yager R R 1981 A procedure for ordering fuzzy subset of the unit subsets of the unit interval. Inform. Sci. 24: $143-161$

Yang L and Liu L 2007 Fuzzy fixed charge solid transportation problem and algorithm. Appl. Soft Computing 7: 879-889

Zadeh L A 1965 Fuzzy sets. Information and Control 8: 338-353 